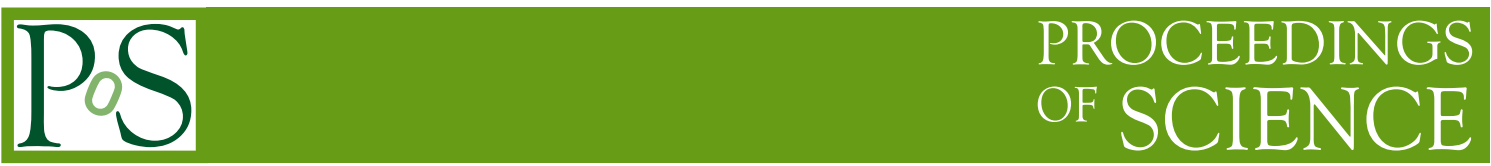

\title{
Chiral Thermodynamics with Charm
}

\author{
Chihiro Sasaki* \\ Frankfurt Institute for Advanced Studies, D-60438 Frankfurt am Main, Germany \\ Institute of Theoretical Physics, University of Wroclaw, PL-50204 Wroclaw, Poland \\ E-mail: sasakiefias.uni-frankfurt.de
}

Chiral thermodynamics of charmed mesons is formulated at finite temperature within a $2+1+1$ flavored effective Lagrangian incorporating heavy quark symmetry. The chiral mass splittings are shown to be less sensitive to the light-quark flavors, attributed to the underlying heavy quark symmetry. Consequently, chiral symmetry restoration is more accelerated in the strange charmedmesons than in the strange light mesons, and this is in striking contrast to the chiral SU(4) result. We also show the correlations between light and heavy flavors around the chiral crossover.

9th International Workshop on Critical Point and Onset of Deconfinement 17-21 November, 2014

ZiF (Center of Interdisciplinary Research), University of Bielefeld, Germany

${ }^{*}$ Speaker. 


\section{Introduction}

Heavy flavors are produced at the initial stage of the high-energy heavy-ion collisions, so that they are expected to carry the dynamical history of a created matter, the Quark-Gluon Plasma (QGP). Recent experimental observations have revealed that charm quarks are thermalized [1, 2, $3,4]$, contrary to earlier anticipation. Charge fluctuations calculated in lattice QCD also indicate that the charmed mesons are deconfined together with light-flavor mesons [5]. Given those observations, comprehensive exploration for the chiral aspects of the heavy-light hadrons increases its importance.

In constructing effective Lagrangians for the heavy-light mesons, besides spontaneous chiral symmetry breaking, heavy quark symmetry is a vital ingredient [6]. The pseudo-scalar $D$ and vector $D^{*}$ states fill in the same multiplet $H$, forming the lowest spin partners. Their low-energy dynamics is dominated by interactions with Nambu-Goldstone (NG) bosons, pions [7, 8, 9, 10]. Introducing the multiplet including $D$ and $D^{*}$ inevitably accompanies another multiplet $G$ which contains a scalar $D_{0}^{*}$ and axial-vector $D_{1}$ states. Those parity partners, $H$ and $G$, become degenerate when the chiral symmetry is restored [11, 12].

Aside from the chiral $S U(4)$ approach where the charm sector suffers from a huge explicit breaking of the extended flavor symmetry, a self-consistent study for the thermal charmed-mesons with implementing heavy quark symmetry has received little attention. In Ref. [13], a chiral effective theory for the light and heavy-light mesons has been formulated in the presence of a medium. Below, we will briefly discuss in-medium masses of the heavy-light mesons and the correlations among light and heavy flavors near the chiral crossover.

\section{Chiral mass splittings}

When the charmed-meson mean fields are introduced to a chiral effective theory in the standard fashion, they act as an extra source which breaks the chiral symmetry explicitly. Consequently, an unrealistically strong mixing between the light-flavor and the charmed meson sector is induced. This defect can be avoided if effective interactions depending on temperature are introduced. Those intrinsic modifications can be extracted from the chiral condensates calculated in lattice QCD. The obtained coupling of the strange charmed meson to the sigma meson, $g_{\pi}^{s}(T)$, becomes quenched as temperature is increased toward the chiral pseudo-critical point $T_{\mathrm{pc}}=154 \mathrm{MeV}$.

Effective charmed-meson masses in hot matter are shown in Fig. 1. The parity partners approach each other as temperature is increased both in the non-strange and strange sector, in consistent with the chiral restoration. The two pseudo-scalar states have the same trend that their masses are increasing with temperature, although the non-strange meson mass exhibits a rather weak modification. On the other hand, the two scalar states drop significantly; the non-strange meson mass by $\sim 200 \mathrm{MeV}$ and the strange meson mass by $\sim 100 \mathrm{MeV}$. The mass splittings between the nonstrange and strange states are around $200 \mathrm{MeV}$ above $T_{\mathrm{pc}}$ due to the fact that the chiral symmetry in the strange sector is not restored yet. Nevertheless, the chiral mass splittings between the scalar and pseudo-scalar states are almost of the same size,

$$
\delta M_{D}\left(T_{\mathrm{pc}}\right) \sim \delta M_{D_{s}}\left(T_{\mathrm{pc}}\right) \sim 200 \mathrm{MeV},
$$



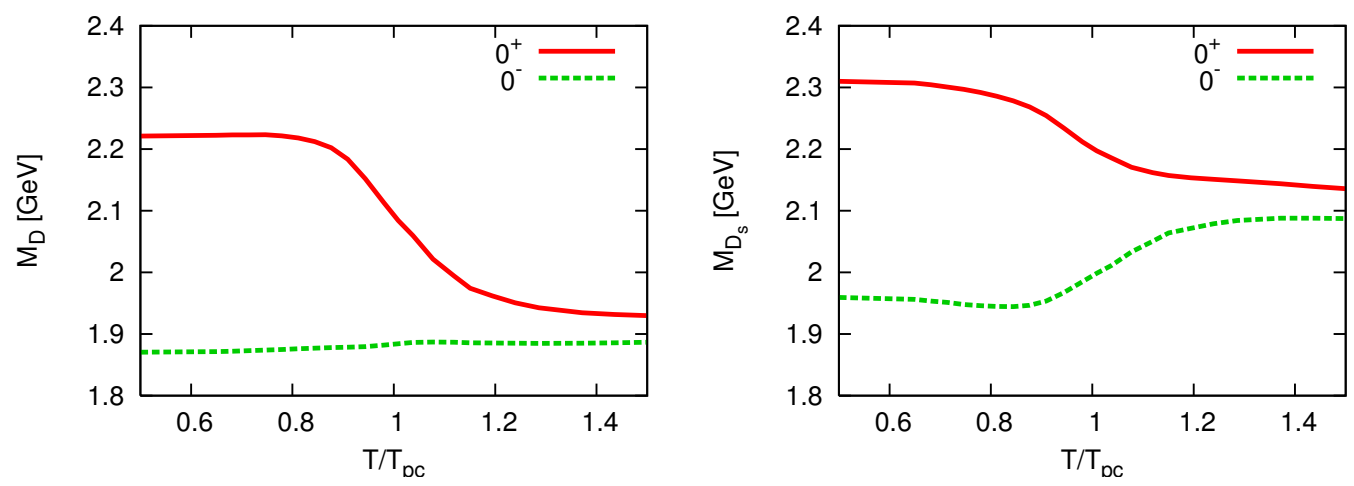

Figure 1: In-medium masses of the non-strange (left) and strange (right) charmed-mesons with positive and negative parity [13].

i.e. the chiral mass differences in the heavy-light sector are blind to the light flavors. This is a striking difference from the chiral properties of the light mesons, and is attributed to the heavy quark symmetry possessed by the leading-order Lagrangian in $1 / m_{Q}$ expansion. In contrast, the chiral SU(4) model, where the charmed mesons are treated on the equal footing to the non-strange and strange mesons, yields a qualitatively different result from Eq. (2.1); $\delta M_{D}$ is much smaller than $\delta M_{D_{s}}$, similar to the light meson masses [14].

The quenched $g_{\pi}^{s}(T)$ leads also to a strong suppression of the scalar $D_{s}$ decay toward $T_{\mathrm{pc}}$, on top of the suppression due to the small isospin violation. Thus, such an anomalous suppression, if it would be observed, is a signature of chiral symmetry restoration. The same should be carried over to the $B$ and $B_{s}$ mesons with which the heavy quark symmetry is more reliable.

\section{Flavor correlations}

Within the same theoretical framework, thermal fluctuations and correlations between the light and heavy-light mesons can also be studied. The extended susceptibility is given in the following matrix form [15]:

$$
\begin{aligned}
& \hat{\chi}_{\sigma \sigma}=\hat{\chi}_{\mathrm{ch}}+\hat{\chi}_{\mathrm{ch}} \hat{\mathscr{C}}_{\mathrm{HL}} \hat{\chi}_{D} \hat{\mathscr{C}}_{\mathrm{HL}} \hat{\chi}_{\mathrm{ch}}, \\
& \hat{\chi}_{\sigma D}=-\hat{\chi}_{\mathrm{ch}} \hat{\mathscr{C}}_{\mathrm{HL}} \hat{\chi}_{D}, \\
& \hat{\chi}_{D \sigma}=-\hat{\chi}_{D} \hat{\mathscr{C}}_{\mathrm{HL}} \hat{\chi}_{\mathrm{ch}}, \\
& \hat{\chi}_{D D}=\hat{\mathscr{C}}_{D}-\hat{\mathscr{C}}_{\mathrm{HL}} \hat{\chi}_{\mathrm{ch}} \hat{\mathscr{C}}_{\mathrm{HL}} \equiv \hat{\chi}_{D},
\end{aligned}
$$

where $\hat{\chi}_{\mathrm{ch}}$ is the chiral susceptibilities of non-strange and strange quark condensates which are responsible for the critical behaviors, and $\hat{\mathscr{C}}_{\mathrm{HL}, D}$ represents the curvature of the effective potential.

Correlations between the light and heavy-light mesons, as well as those between the heavylight mesons, are shown in Fig. 2. Due to the embedding of $\hat{\chi}_{\mathrm{ch}}$, various heavy-light flavor correlations are modified qualitatively in a narrow range of temperature where the chiral susceptibility exhibits a peak. The onset of the chiral crossover, in the heavy-light flavor correlations, is therefore independent from the light flavors. This indicates that the fluctuations carried by strange charmed 

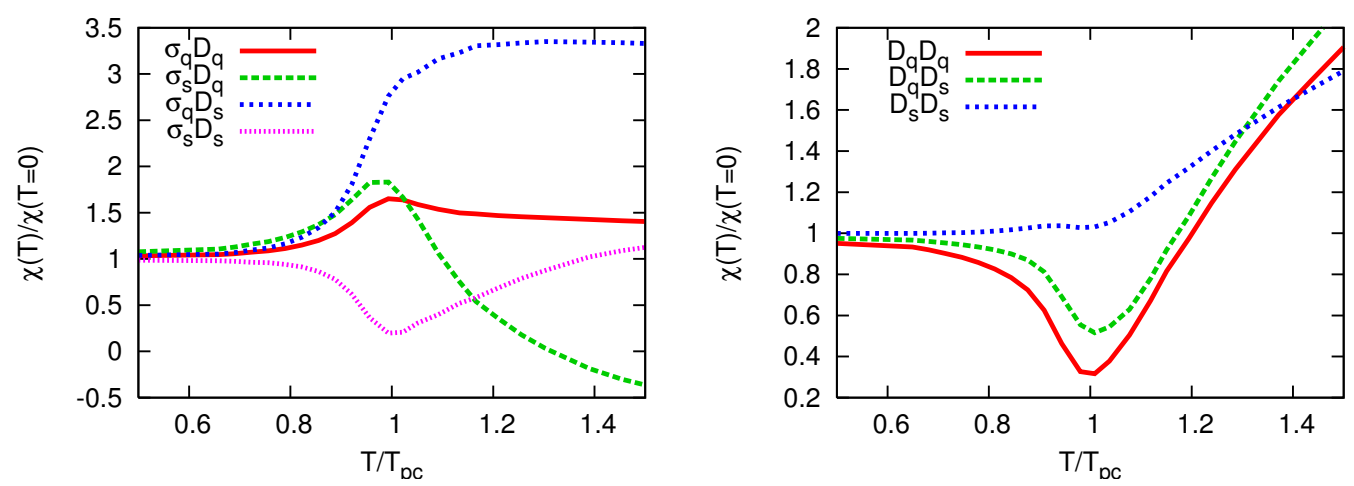

Figure 2: Flavor correlations between the light and heavy-light mesons (left) and among heavy-light mesons (right).

mesons can also be used to identify the chiral crossover, which is dominated by the non-strange light quark dynamics.

\section{Conclusions}

The chiral mass splittings are shown to be essentially insensitive to the light-quark flavors, in spite of a non-negligible explicit breaking of the chiral $S U(3)$ symmetry. This "blindness" of the charm quark to the light degrees of freedom is dictated by the heavy quark symmetry. In contrast, the kaon and its chiral partner masses become degenerate at a higher temperature than $T_{\mathrm{pc}}$, indicating a delay of the $S U(3)$ symmetry restoration. In the heavy-light sector, on the other hand, the strange charmed meson captures the onset of chiral symmetry restoration more strongly than the strange light meson does. A similar consequence is found in the correlations involving the heavy-light meson mean fields. Those fluctuations exhibit certain qualitative changes around $T_{\mathrm{pc}}$, and this feature is independent of the light flavors. Hence, the strange charmed mesons have a potential for measuring a remnant of $O(4)$.

It is a real challenge to explore the thermodynamics of heavy-light hadrons at high density. A central task is to introduce a reliable density dependence of the interaction parameters, which requires either a more microscopic prescription in the effective theory side, or more precise data for the chiral condensates from lattice simulations. There are some implications of a confined phase with unbroken chiral symmetry from the lattice studies where the low-lying Dirac modes, which generate a non-vanishing chiral condensate via the Banks-Casher relation, are removed. This new state of hadronic matter might appear in the QCD phase diagram at high baryon density, where chiral hadronic theories may become valid in a wider parameter range (See Ref. [16] and references therein).

\section{Acknowledgments}

The work has been partly supported by the Hessian LOEWE initiative through the Helmholtz International Center for FAIR (HIC for FAIR), and by the Polish Science Foundation (NCN) under Maestro grant DEC-2013/10/A/ST2/00106. 


\section{References}

[1] A. Adare et al. [PHENIX Collaboration], Phys. Rev. C 84, 044905 (2011).

[2] L. Adamczyk et al. [STAR Collaboration], arXiv:1404.6185 [nucl-ex].

[3] B. Abelev et al. [ALICE Collaboration], JHEP 1209, 112 (2012).

[4] B. Abelev et al. [ALICE Collaboration], Phys. Rev. Lett. 111, 102301 (2013).

[5] A. Bazavov, H.-T. Ding, P. Hegde, O. Kaczmarek, F. Karsch, E. Laermann, Y. Maezawa and S. Mukherjee et al., Phys. Lett. B 737, 210 (2014).

[6] N. Isgur and M. B. Wise, Phys. Lett. B 232, 113 (1989); Phys. Lett. B 237, 527 (1990).

[7] H. Georgi, Phys. Lett. B 240, 447 (1990).

[8] M. B. Wise, Phys. Rev. D 45, 2188 (1992).

[9] G. Burdman and J. F. Donoghue, Phys. Lett. B 280, 287 (1992).

[10] T. M. Yan, H. Y. Cheng, C. Y. Cheung, G. L. Lin, Y. C. Lin and H. L. Yu, Phys. Rev. D 46, 1148 (1992) [Erratum-ibid. D 55, 5851 (1997)].

[11] M. A. Nowak, M. Rho and I. Zahed, Phys. Rev. D 48, 4370 (1993).

[12] W. A. Bardeen and C. T. Hill, Phys. Rev. D 49, 409 (1994).

[13] C. Sasaki, Phys. Rev. D 90, no. 11, 114007 (2014).

[14] D. Roder, J. Ruppert and D. H. Rischke, Phys. Rev. D 68, 016003 (2003).

[15] C. Sasaki and K. Redlich, arXiv:1412.7365 [hep-ph].

[16] C. Sasaki, Nucl. Phys. A 931, 238 (2014). 